\title{
Plasma tenofovir trough concentrations are associated with renal dysfunction in Japanese patients with HIV infection: a retrospective cohort study
}

Yusuke Kunimoto ${ }^{1 *}$, Hiroshi lkeda ${ }^{2}$, Satoshi Fujii ${ }^{1}$, Manabu Kitagawa ${ }^{1}$, Kieko Yamazaki ${ }^{1}$, Hiromasa Nakata ${ }^{1}$, Norimasa Noda', Tadao Ishida ${ }^{3}$ and Atsushi Miyamoto ${ }^{1}$

\begin{abstract}
Background: Plasma tenofovir (TFV) trough concentrations may be relevant for tenofovir disoproxil fumarate (TDF)-induced renal dysfunction. The purpose of this study was to determine the association between plasma TFV trough concentrations and TDF-induced renal dysfunction in Japanese patients with human immunodeficiency virus (HIV) infection.

Methods: A 48-week, retrospective cohort study was performed with Japanese patients with HIV infection who started a TDF-containing combination antiretroviral therapy regimen. Plasma TFV trough concentrations were obtained at steady state. The following variables were included in the analysis: sex, age, body weight, body mass index (BMI), serum creatinine, CD4+ cell count, HIV-RNA, concomitant medications, comorbidities, plasma TFV trough concentrations, and estimated glomerular filtration rate (eGFR). For comparisons of variables, we used Mann-Whitney $U$ tests or Fisher's exact tests. Then, variables associated with renal dysfunction in the univariate analysis were entered into correlation analysis.
\end{abstract}

Results: The analysis included 11 patients. The rate of decrease in eGFR was significantly correlated with body weight (Spearman correlation $=-0.645, p=0.041$ ), BMI (Spearman correlation $=-0.682, p=0.031$ ), and plasma TFV trough concentrations (Spearman correlation $=0.709, p=0.025$ ).

Conclusions: Despite the small sample size, our findings suggest that higher plasma TFV trough concentrations may cause TDF-induced renal dysfunction. To prevent TDF-induced renal dysfunction, we propose that individual monitoring of plasma TFV trough concentrations should be performed in Japanese patients with HIV infection.

Keywords: Combination antiretroviral therapy, HIV, Plasma tenofovir trough concentration, Renal dysfunction, Tenofovir disoproxil fumarate, Therapeutic drug monitoring

\section{Background}

Tenofovir disoproxil fumarate (TDF) belongs to the nucleotide reverse transcriptase inhibitor (NRTI) class of antiretroviral drugs (ARVs) and is a preferred component of first-line regimens in the US Department of Health and Human Services guidelines [1]. Therefore, promoting safe use of TDF is important during the

\footnotetext{
* Correspondence: kunimoto@sapmed.ac.jp

'Department of Hospital Pharmacy, Sapporo Medical University Hospital,

South 1, West 16, Chuo-ku, Sapporo, Hokkaido 060-8543, Japan

Full list of author information is available at the end of the article
}

management of long-term combination antiretroviral therapy (cART). Although TDF has a good safety profile, TDF-induced renal dysfunction is a well-known adverse effect $[2,3]$.

The most notable adverse effect with TDF is renal dysfunction, and one study reported that the incidence of TDF-induced renal dysfunction was high in Japanese patients [4]. TDF is rapidly converted to tenofovir (TFV) following absorption, and plasma TFV trough concentrations may be relevant for TDF-induced renal dysfunction. High plasma TFV trough concentrations are 
reportedly associated with a decrease in glomerular function [5]. Furthermore, several studies conducted in Japan reported that low body weight and genetic polymorphisms of drug transporter genes are significantly associated with TDF-induced renal dysfunction $[4,6]$. However, reports of the association between plasma TFV trough concentrations and TDF-induced renal dysfunction in Japanese patients with human immunodeficiency virus (HIV) infection are lacking. Moreover, the effect of plasma TFV trough concentrations on renal dysfunction is not guaranteed to be the same between Japanese patients and patients of other ethnic groups. For safe use of TDF in Japan, the risk factors for TDFinduced renal dysfunction in Japanese patients need to be understood.

Therapeutic drug monitoring (TDM) is an important clinical technique for personalized medicine. In ART, TDM is recommended in specific clinical scenarios, such as for cases in which pathophysiological changes (e.g., renal dysfunction) and/or drug-drug interactions adversely affect pharmacokinetics [1, 7]. However, TDM for cART is not recommended for routine use in the clinical care of HIV-infected patients because of a lack of large prospective studies showing that TDM improves clinical or virologic outcomes [1]. We anticipated that clarifying the usefulness of monitoring plasma TFV trough concentrations could prevent TDF-induced renal dysfunction.

Therefore, the purpose of this study was to determine the association between plasma TFV trough concentrations and TDF-induced renal dysfunction.

\section{Methods}

\section{Patients}

A 48-week, retrospective cohort study was performed in Sapporo Medical University Hospital. Data for HIVinfected patients who started a TDF-containing cART regimen between August 2008 and October 2013 were collected from the medical records. Patients who received cART with TDF for at least 2 weeks were enrolled in this exploratory study. Patients with renal disease that could affect renal function were excluded from the analysis. All the patients provided written informed consent before enrollment, and the protocol was approved (25152 ) by the ethical review board of Sapporo Medical University.

\section{Measurements}

Patients with plasma TFV trough concentration measurements performed at steady state $\geq 21 \mathrm{~h}$ after drug intake were included in the analysis. Information about the time of last drug intake was reported at the time of sample collection. Pre-dose blood samples were collected in heparinized tubes and centrifuged for $10 \mathrm{~min}$ at $3000 \mathrm{rpm}$; the resultant plasma was removed and stored at $-20{ }^{\circ} \mathrm{C}$ until analysis. Plasma TFV concentrations were analyzed using high-performance liquid chromatography, and fluorescence was detected at $254 \mathrm{~nm}$ for excitation and $425 \mathrm{~nm}$ for emission (BML Inc., Japan). Estimated glomerular filtration rate (eGFR) was calculated using the Japanese equation that was developed by the Japanese Society of Nephrology, eGFR = $194 \times[\text { serum creatinine }]^{-1.094} \times[\text { age }]^{-0.287} \times[0.739$ for women] [8]. Renal function was classified as follows: stage 1 , eGFR $\geq 90 \mathrm{~mL} / \mathrm{min} \cdot 1.73 \mathrm{~m}^{2}$; stage $2,90>$ eGFR $\geq 60 \mathrm{~mL} / \mathrm{min} \cdot 1.73 \mathrm{~m}^{2}$; stage $3,60>$ eGFR $\geq$ $30 \mathrm{~mL} / \mathrm{min} \cdot 1.73 \mathrm{~m}^{2}$; stage $4,30>\mathrm{eGFR} \geq 15 \mathrm{~mL} / \mathrm{min}$. $1.73 \mathrm{~m}^{2}$; and stage 5 , eGFR $<15 \mathrm{~mL} / \mathrm{min} \cdot 1.73 \mathrm{~m}^{2}$ [9]. Renal dysfunction was defined based on the progression of classified chronic kidney disease (CKD) stages after treatment [10]. The rate of decrease in eGFR was

Table 1 Patient characteristics at baseline

\begin{tabular}{|c|c|}
\hline & $\begin{array}{l}\text { Study patients } \\
N=11\end{array}$ \\
\hline Male sex, n (\%) & $11(100)$ \\
\hline Age (years) ${ }^{a}$ & $35(32-45)$ \\
\hline Body weight $(\mathrm{kg})^{a}$ & $56.1(51.6-67.3)$ \\
\hline $\mathrm{BMI}\left(\mathrm{kg} / \mathrm{m}^{2}\right)^{\mathrm{a}}$ & $18.2(17.6-22.8)$ \\
\hline $\mathrm{BSA}\left(\mathrm{m}^{2}\right)$ & $1.69(1.60-1.79)$ \\
\hline Serum creatinine $(\mathrm{mg} / \mathrm{dL})^{a}$ & $0.80(0.70-0.80)$ \\
\hline eGFR $\left(\mathrm{mL} / \mathrm{min} \cdot 1.73 \mathrm{~m}^{2}\right)^{a}$ & $96.1(81.9-103.1)$ \\
\hline \multicolumn{2}{|l|}{ CKD stage, n (\%) } \\
\hline 1 & $7(63.6)$ \\
\hline 2 & $4(36.4)$ \\
\hline 3 & $0(0)$ \\
\hline 4 & $0(0)$ \\
\hline 5 & $0(0)$ \\
\hline $\mathrm{CD}^{+}$cell count $(/ \mu \mathrm{L})^{\mathrm{a}}$ & $119(37-211)$ \\
\hline HIV RNA viral load $\left(\log _{10} / \mathrm{mL}\right)^{a}$ & $4.83(4.61-5.85)$ \\
\hline Protease inhibitors (ritonavir-boosted), n (\%) & $7(63.6)$ \\
\hline Protease inhibitors (unboosted), n (\%) & $0(0)$ \\
\hline NNRTIs, n (\%) & $0(0)$ \\
\hline INSTIS, n (\%) & $4(36.4)$ \\
\hline Concurrent use of a nephrotoxic drug, n (\%) & $4(36.4)$ \\
\hline Hypertension, n (\%) & $0(0)$ \\
\hline Diabetes, n (\%) & $0(0)$ \\
\hline Hepatitis B, n (\%) & $3(27.3)$ \\
\hline Hepatitis C, n (\%) & $0(0)$ \\
\hline Plasma TFV concentration $(\mathrm{ng} / \mathrm{mL})^{a}$ & $64.0(56.0-90.5)$ \\
\hline
\end{tabular}

$B M I$ body mass index, BSA body surface area, eGFR estimated glomerular filtration rate, $C K D$ chronic kidney disease, NNRTIs non-nucleoside reversetranscriptase inhibitors, INSTIs integrase strand transfer inhibitors, TFV tenofovir ${ }^{a}$ Values are reported as median (interquartile range) 
defined as the maximum rate of decrease in eGFR from baseline during the study period.

Other variables included in the analysis were sex, age, body weight, body mass index (BMI), body surface area (BSA), serum creatinine concentration, CD4+ cell count, HIV-RNA, concomitant medications, comorbidities, and plasma TFV trough concentrations.

\section{Statistical analysis}

The time from baseline to renal dysfunction was analyzed using the Kaplan-Meier method. Baseline characteristics were compared between patients with and without renal dysfunction using Mann-Whitney $U$ tests or Fisher's exact tests. Univariate analysis was conducted to reduce the list of potential variables associated with renal dysfunction. The potential variables associated with renal dysfunction with $p \leq 0.1$ in the univariate analysis were entered into correlation analyses. Statistical significance was defined as a two-sided $p<0.05$. All analyses were performed using StatMate IV (ATMS Co. Ltd., Tokyo, Japan).

\section{Results}

Of the 12 patients who were enrolled, one patient was excluded due to a congenital solitary kidney. Patient characteristics are presented in Table 1 . All of the study patients were men and had a relatively low body weight (median body weight, $56.1 \mathrm{~kg}$ ). No patient had comorbidities such as hypertension, diabetes, or hepatitis C. The cART regimens included a protease inhibitor for $63.6 \%$ of the patients $(n=5$, darunavir/ritonavir; $n=2$, fosamprenavir/ritonavir) and an integrase strand transfer inhibitor (raltegravir) for $36.4 \%$ of the patients. The median time to plasma TFV trough concentration measurements was 9 weeks after starting a TDF-containing cART regimen.

For the seven patients (63.6 \%) in whom renal dysfunction occurred, it occurred within 24 weeks from cART initiation. The cumulative incidence of renal dysfunction in these patients was 9.1, 27.3, 45.5, 54.5, and $63.6 \%$ at $4,8,12,16-20$, and $24-48$ weeks, respectively. The changes in classified CKD stage after treatment in these patients were from stage 1 to 2 , stage 1 to 3 , and stage 2 to 3 in 3, 2, and two patients, respectively.

Table 2 compares the baseline characteristics between patients with and without renal dysfunction. The rate of decrease in eGFR was significantly different between the 2 groups $(p=0.008)$. In patients with renal dysfunction, age and plasma TFV trough concentrations tended to be higher, and weight and BMI tended to be lower. Data regarding the 11 patients are given in Additional file 1.

The rate of decrease in eGFR was not significantly correlated with age (Fig. 1a, Spearman correlation $=0.403$, $p=0.203$ ), but was significantly correlated with weight (Fig. 1b, Spearman correlation $=-0.645, p=0.041$ ), BMI (Fig. 1c, Spearman correlation $=-0.682, p=0.031$ ), and plasma TFV trough concentrations (Fig. 1d, Spearman correlation $=0.709, p=0.025$ ).

\section{Discussion}

We showed that higher plasma TFV trough concentrations tended to be associated with a decrease in eGFR after starting cART. In principle, ARVs are required for a person's entire lifetime. Most of the NRTI class of

Table 2 Characteristics of patients with or without renal dysfunction

\begin{tabular}{|c|c|c|c|}
\hline & $\begin{array}{l}\text { Renal dysfunction patients } \\
N=7\end{array}$ & $\begin{array}{l}\text { Non-renal dysfunction patients } \\
N=4\end{array}$ & $p$ value \\
\hline Age (years) ${ }^{a}$ & $45.0(38.0-52.0)$ & $29.5(24.0-35.8)$ & 0.072 \\
\hline Body weight $(\mathrm{kg})^{\text {a }}$ & $52.2(49.4-62.9)$ & $60.8(59.0-68.8)$ & 0.078 \\
\hline $\mathrm{BMI}\left(\mathrm{kg} / \mathrm{m}^{2}\right)^{\mathrm{a}}$ & $17.7(17.2-20.9)$ & $21.3(20.2-23.8)$ & 0.078 \\
\hline $\operatorname{BSA}\left(\mathrm{m}^{2}\right)^{\mathrm{a}}$ & $1.60(1.57-1.77)$ & $1.70(1.69-1.79)$ & 0.169 \\
\hline Serum creatinine $(\mathrm{mg} / \mathrm{dL})^{\text {a }}$ & $0.70(0.65-0.80)$ & $0.80(0.78-0.83)$ & 0.163 \\
\hline $\mathrm{eGFR}\left(\mathrm{mL} / \mathrm{min} \cdot 1.73 \mathrm{~m}^{2}\right)^{\mathrm{a}}$ & $96.1(85.9-102.9)$ & $90.4(82.7-104.2)$ & 0.925 \\
\hline Rate of decrease in eGFR (\%) ${ }^{a}$ & $33.2(24.3-38.2)$ & $11.7(8.0-12.9)$ & 0.008 \\
\hline $\mathrm{CD}^{+}$cell count $(/ \mu \mathrm{L})^{\mathrm{a}}$ & $58(36-133)$ & $204(99-320)$ & 0.149 \\
\hline HIV RNA viral load $\left(\log _{10} / \mathrm{mL}\right)^{a}$ & $4.63(4.52-5.84)$ & $5.33(4.82-5.92)$ & 0.395 \\
\hline Protease inhibitors (ritonavir-boosted), n (\%) & $4(57.1)$ & $3(75.0)$ & 1.000 \\
\hline INSTIS, n (\%) & $3(42.9)$ & $1(25.0)$ & 1.000 \\
\hline Concurrent use of a nephrotoxic drug, n (\%) & $2(28.6)$ & $2(50)$ & 0.576 \\
\hline Hepatitis B, n (\%) & $3(42.6)$ & $0(0)$ & 0.236 \\
\hline Plasma TFV concentration $(\mathrm{ng} / \mathrm{mL})^{a}$ & $88.0(67.0-102.5)$ & $56.0(50.5-60.5)$ & 0.073 \\
\hline
\end{tabular}

$B M I$ body mass index, BSA body surface area, eGFR estimated glomerular filtration rate, INSTIs integrase strand transfer inhibitors, TFV tenofovir ${ }^{a}$ Values are reported as median (interquartile range) 

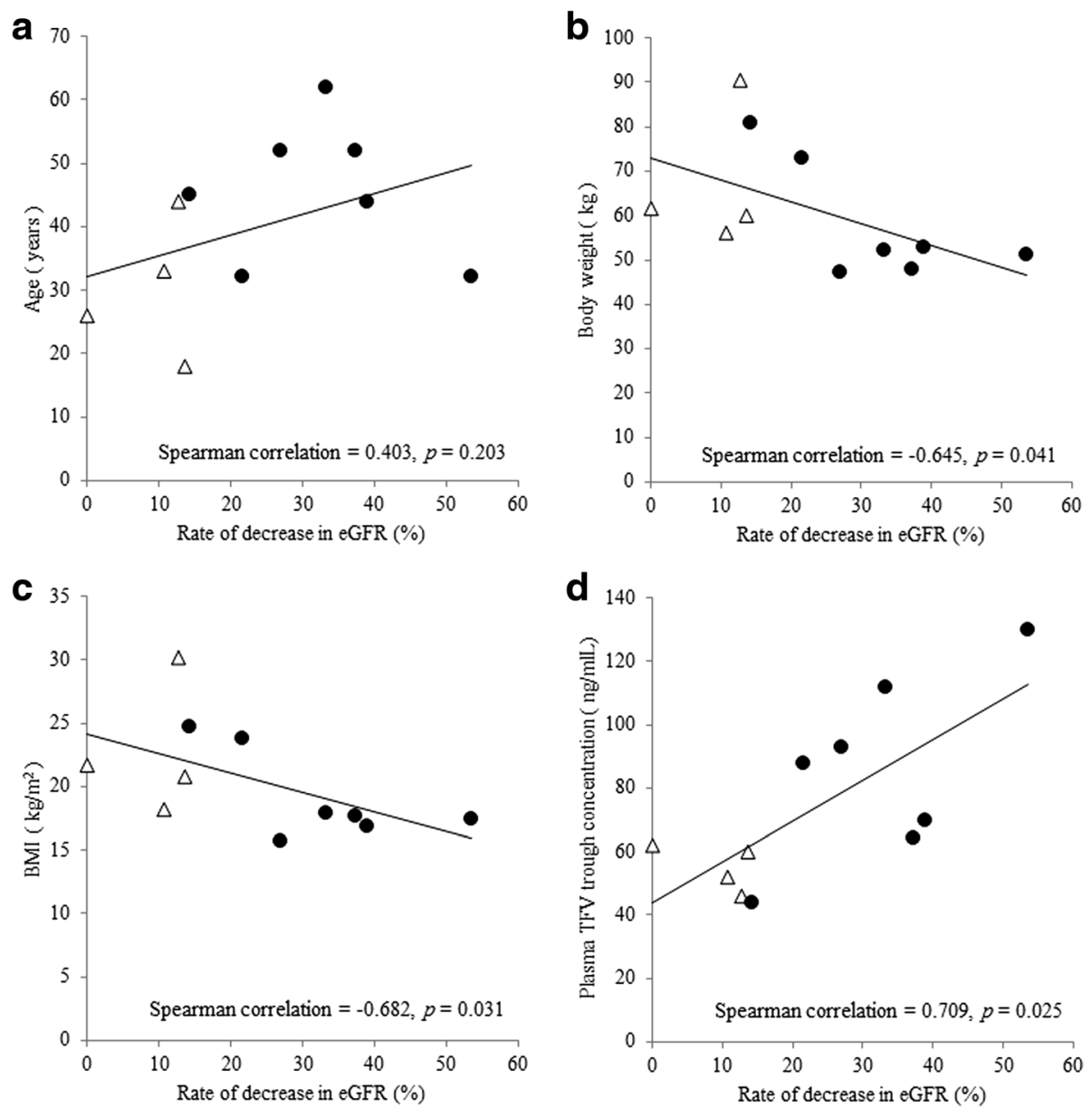

Fig. 1 Correlation analysis between eGFR decrease rate and potential variables associated with renal dysfunction. Patients with renal dysfunction are indicated by black circles, and patients without renal dysfunction are indicated by white triangles. eGFR, estimated glomerular filtration rate; BMI, body mass index; TFV, tenofovir

ARVs that are used as first-line regimens are of the renal excretion type. Therefore, choosing ARVs for patients with renal dysfunction is often difficult. Based on our results, we propose individual monitoring of plasma TFV trough concentrations in Japanese patients to prevent TDF-induced renal dysfunction.

Our data indicated that special attention should be given to TDF-induced renal dysfunction within 24 weeks from cART initiation. Not only did renal dysfunction develop within 24 weeks for all 7 patients in the present study, eGFR rapidly decreased in a group administered TDF during the first 24 weeks in a previous study [3]. Therefore, monitoring of renal function should be primarily conducted during the first 24 weeks after initiating a TDF-containing cART regimen.

Plasma TFV trough concentrations showed a stronger correlation with the rate of decrease in eGFR than the other variables, suggesting that plasma TFV trough concentrations are useful to predict the occurrence of TDF-induced renal dysfunction. Additionally, the rate of decrease in
eGFR uncorrected for BSA was also significantly correlated with the plasma TFV trough concentration (Spearman correlation $=0.709, p \leq 0.025$ ). Furthermore, in the analysis of the data for the seven patients without concurrent use of a nephrotoxic drug, the rate of decrease in eGFR was significantly correlated with plasma TFV trough concentrations (Spearman correlation $=0.900, p<0.011$ ). The significant correlation between the rate of decrease in eGFR and plasma TFV trough concentrations supports the findings of a previous study in which high plasma TFV trough concentrations were associated with a decrease in eGFR [5]; the median decrease in eGFR at 12 months after cART initiation was $8.5 \mathrm{~mL} / \mathrm{min}$ in the group with high plasma TFV trough concentrations $(>90 \mathrm{ng} / \mathrm{mL})$. In the present study, three patients had high plasma TFV trough concentrations ( $>90 \mathrm{ng} / \mathrm{mL}$ ), with decreases in eGFR at 48 weeks after cART initiation of $22.3,29.4$, and $57.2 \mathrm{~mL} / \mathrm{min}$. Therefore, the impact of higher plasma TFV trough concentrations on eGFR is greater in Japanese patients. In addition, the occurrence of TDF-induced renal dysfunction might be predicted 
from higher plasma TFV trough concentrations in Japanese patients. The rate of decrease in eGFR and body weight or BMI was also significantly and negatively correlated, suggesting that the development of TDF-induced renal dysfunction is affected by low body weight and BMI. Low body weight was also associated with TDF-induced renal dysfunction in a previous report [4], and plasma TFV concentrations can be affected by low body weight, genetic polymorphisms of drug transporter genes, and other factors [11]. Additionally, in a study with women, decreasing BMI and other factors were associated with elevated TFV exposure [12]. When measurement of plasma TFV trough concentrations for individual monitoring purposes in Japanese patients is not possible, we suggest that low body weight or low BMI could serve as a reasonable alternative predictor of TDF-induced renal dysfunction.

Our study has several limitations. First, the main limitation of our study is the small sample size. Future studies are needed to replicate our results with a larger sample size. Second, formulations containing TDF are only available in oral tablets, and powder formulations for the purposes of dose adjustment do not exist in Japan. To reduce the single dose amount and extend the dosing interval (e.g., every $48 \mathrm{~h}$ ), crushed tablets could be used to control higher plasma TFV trough concentrations. However, this could cause issues with maintaining medication adherence. Therefore, reducing the dose of TDF based on TDM is not currently easy.

\section{Conclusions}

In conclusion, this study showed that higher plasma TFV trough concentrations are associated with a decrease in eGFR in Japanese patients, and plasma TFV trough concentrations might predict the occurrence of TDF-induced renal dysfunction. In the future, to prevent TDF-induced renal dysfunction, reduction of the TDF dose or a switch from TDF to a different ARV could be considered with high plasma TFV trough concentrations. Although more studies are needed to identify the factors related with TDF-induced renal dysfunction, we believe that the use of plasma TFV trough concentrations could help prevent this adverse event.

\section{Additional file}

Additional file 1 Data regarding the 11 patients. (XLSX $10 \mathrm{~kb})$

\section{Abbreviations}

ARV: Antiretroviral drug; BMI: Body mass index; CART: Combination antiretroviral therapy; eGFR: Estimated glomerular filtration rate; HIV: Human immunodeficiency virus; NRTI: Nucleotide reverse transcriptase inhibitor; TDF: Tenofovir disoproxil fumarate; TDM: Therapeutic drug monitoring; TFV: Tenofovir

Acknowledgments

Not applicable.

\section{Funding}

This work was supported in part by a Grant-in-Aid for Scientific Research from the Japan Society for the Promotion of Science.

\section{Availability of data and materials}

The dataset supporting the conclusions of this article is included within the article and its additional file.

\section{Authors' contributions}

YK designed this concept originally, performed the analytical experiments and statistical analyses, and wrote the manuscript. $\mathrm{HI}$ and $\mathrm{TI}$ acquired the informed consent and performed the clinical evaluations of the patients. SF, MK, KY, HN, NN, and AM interpreted and discussed the data. All authors have read and approved the final manuscript.

\section{Competing interests}

The authors declare that they have no competing interests.

\section{Ethical approval and consent to participate}

Patients provided informed consent before enrollment, and the protocol was conducted with permission (25-152) from the Sapporo Medical University ethical review board.

\section{Author details}

${ }^{1}$ Department of Hospital Pharmacy, Sapporo Medical University Hospital, South 1, West 16, Chuo-ku, Sapporo, Hokkaido 060-8543, Japan. ²Department of Gastroenterology, Rheumatology and Clinical Immunology, Sapporo Medical University School of Medicine, South 1, West 16, Chuo-ku, Sapporo, Hokkaido 060-8543, Japan. ${ }^{3}$ Department of Hematology, Japanese Red Cross Medical Center, 4-1-22 Hiroo, Shibuya-ku, Tokyo 150-8935, Japan.

Received: 30 April 2016 Accepted: 14 September 2016

Published online: 22 September 2016

\section{References}

1. Panel on Antiretroviral Guidelines for Adults and Adolescents. Guidelines for the use of antiretroviral agents in HIV-1-infected adults and adolescents. Department of Health and Human Services. 2016. https://aidsinfo.nih.gov/ contentfiles/AdultandAdolescentGL.pdf. Accessed 26 Feb 2016.

2. Schaaf B, Aries SP, Kramme E, Steinhoff J, Dalhoff K. Acute renal failure associated with tenofovir treatment in a patient with acquired immunodeficiency syndrome. Clin Infect Dis. 2003;37:e41-3.

3. Nishijima T, Gatanaga H, Komatsu H, Tsukada K, Shimbo T, Aoki T, et al. Renal function declines more in tenofovir- than abacavir-based antiretroviral therapy in low-body weight treatment-naive patients with HIV infection. PLoS One. 2012:7:e29977.

4. Nishijima T, Komatsu H, Gatanaga H, Aoki T, Watanabe K, Kinai E, et al. Impact of small body weight on tenofovir-associated renal dysfunction in HIV-infected patients: a retrospective cohort study of Japanese patients. PLoS One. 2011;6:e22661.

5. Poizot-Martin I, Solas C, Allemand J, Obry-Roguet V, Pradel V, Bregigeon S, et al. Renal impairment in patients receiving a tenofovir-CART regimen: impact of tenofovir trough concentration. J Acquir Immune Defic Syndr. 2013;62:375-80

6. Nishijima T, Komatsu H, Higasa K, Takano M, Tsuchiya K, Hayashida T, et al Single nucleotide polymorphisms in ABCC2 associate with tenofovirinduced kidney tubular dysfunction in Japanese patients with HIV-1 infection: a pharmacogenetic study. Clin Infect Dis. 2012;55:1558-67.

7. Kunimoto $Y$, Yasui H, Touda N, Okazaki M, Nakata H, Noda N, et al. Coadministration of tenofovir decreased atazanavir plasma concentration after unilateral nephrectomy. J Infect Chemother. 2013;19:750-3.

8. Matsuo S, Imai E, Horio M, Yasuda Y, Tomita K, Nitta K, et al. Revised equations for estimated GFR from serum creatinine in Japan. Am J Kidney Dis. 2009;53:982-92.

9. National Kidney Foundation. K/DOQI clinical practice guidelines for chronic kidney disease: evaluation, classification, and stratification. Am J Kidney Dis. 2002;39:S1-266.

10. Fujii S, Takahashi S, Makino S, Kunimoto Y, Nakata H, Noda N, et al. Impac of vancomycin or linezolid therapy on development of renal dysfunction and thrombocytopenia in Japanese patients. Chemotherapy. 2013;59:319-24 
11. Rungtivasuwan $\mathrm{K}$, Avihingsanon $\mathrm{A}$, Thammajaruk N, Mitruk S, Burger DM, Ruxrungtham $\mathrm{K}$, et al. Influence of $A B C C 2$ and $A B C C 4$ polymorphisms on tenofovir plasma concentrations in Thai HIV-infected patients. Antimicrob Agents Chemother. 2015:59:3240-5.

12. Baxi SM, Greenblatt RM, Bacchetti P, Scherzer R, Minkoff H, Huang $Y$, et al. Common clinical conditions-age, low BMl, ritonavir use, mild renal impairment-affect tenofovir pharmacokinetics in a large cohort of HIVinfected women. AIDS. 2014;28:59-66.

Submit your next manuscript to BioMed Central and we will help you at every step:

- We accept pre-submission inquiries

- Our selector tool helps you to find the most relevant journal

- We provide round the clock customer support

- Convenient online submission

- Thorough peer review

- Inclusion in PubMed and all major indexing services

- Maximum visibility for your research

Submit your manuscript at www.biomedcentral.com/submit 